\title{
An uncommon cause of foot ulcer: tuberculous osteomyelitis
}

\author{
M C Yuen, W K Tung
}

\begin{abstract}
Tuberculous osteomyelitis is an uncommon infection that usually involves the vertebrae. An otherwise healthy young man with a chronic discharging sinus on his right foot caused by tuberculous osteomyelitis is described. The risk factors, clinical features, radiological findings, and investigations of tuberculous osteomyelitis are briefly reviewed. Tuberculous osteomyelitis usually runs an insidious course; emergency physicians should be aware of the possibility of tuberculous osteomyelitis especially when patients present with chronic unexplained musculoskeletal symptoms.

(Emerg Med f 2001;18:140-141)
\end{abstract}

Keywords: tuberculosis; osteomyelitis; metatarsal bones

\section{Case report}

A 22 year old man presented to the accident and emergency department for a chronic skin ulcer over his right foot. His past health was good and he worked as a clerk. He neither smoked nor drank. He denied use of illicit drugs and there was no history of trauma. The ulcer appeared about one month ago and no improvement was seen with antibiotics prescribed by his general practitioner. $\mathrm{He}$ also had chronic non-productive cough for one month. There was neither dypsnoea nor chest pain. Constitutional symptoms like fever or weight loss were absent.

Physical examination revealed a $1 \mathrm{~cm}$ skin ulcer overlying the lateral side of his right foot (fig 1). There was mild surrounding cellulitis. The chest was clear on auscultation. Radiographs of chest and right foot were performed. The foot radiograph revealed an osteolytic area with surrounding osteopenia had replaced the head of his 5 th metatarsal bone (fig 2). The chest radiograph showed right upper lobe haziness. A clinical diagnosis of pulmonary tuberculosis with chronic osteomyelitis was made. $\mathrm{He}$ was admitted to the orthopaedic ward for further management.

His complete blood count, liver and renal function tests were normal. Serum for HIV antibodies was negative. The orthopaedic surgeon performed open excisional biopsy of the lesion. The histological examination of the necrotic tissue showed acid fast bacilli with granulomatous inflammation. The culture of the necrotic tissue grew mycobacterium tuberculosis. He was given pyrazinamide, isoniazid and rifampicin. After nine months of treatment, there was clinical and radiological resolution of the pulmonary tuberculosis and osteomyelitis of 5 th metatarsal head.

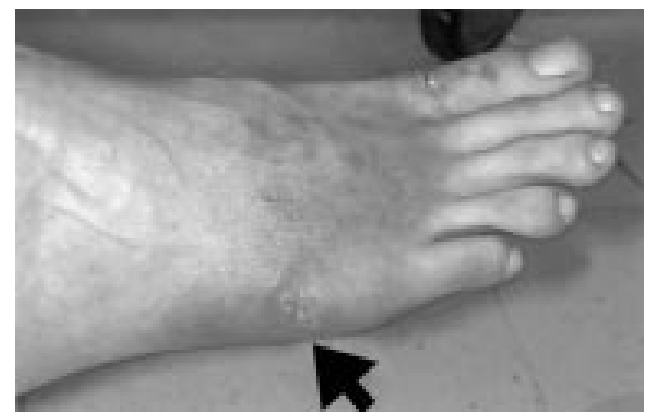

Figure 1 The arrows indicates the small ulcer overlying the right 5 th metatarsal head.

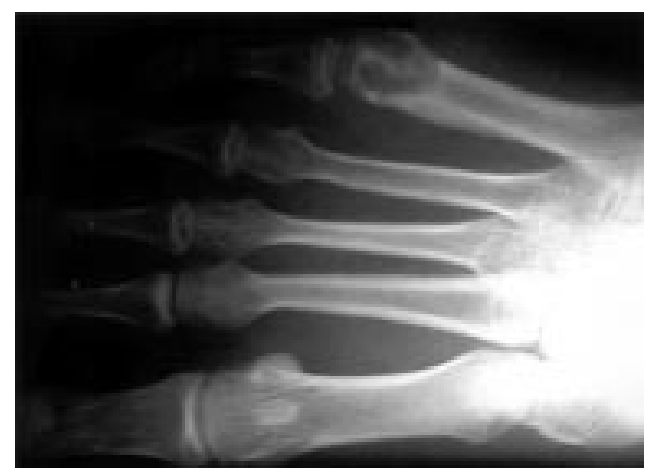

Figure 2 An osteolytic area with surrounding osteopenia replaced the 5 th metatarsal head.

\section{Discussion}

Tuberculosis remains a major health problem in developing as well as developed countries despite the advances in antituberculosis treatment. In fact, the incidence of tuberculosis tends to rise again, which is probably related to the following reasons: appearance of HIV; increasing number of patients treated with immunosuppressive agents; emergence of drug resistant strains of mycobacterium, and an increasing number of healthcare workers exposed to the disease. ${ }^{1}$ Although immunocompromised patients are at risk of tuberculosis, tuberculosis also affects otherwise young healthy populations such as the patient presented.

Tuberculous osteomyelitis and arthritis accounts for $10 \%$ of the cases of extrapulmonary tuberculosis. ${ }^{2}$ Tuberculous osteomyelitis, like other forms of extrapulmonary tuberculosis, is secondary to lymphohematogenous dissemination to bones from the lungs. ${ }^{34}$ Tuberculosis has been reported to affect all the bones in the body. The commonest affected sites are spines, followed by femur, tibia and fibula. ${ }^{3}$ Tuberculosis osteomyelitis of metatarsal bones had been reported, though it is considered as a rare site of involvement. In Vohra's series, 4 of 28 patients had their metatarsal bones involved. ${ }^{1}$
Accepted for publication 19 July 2000 
Usually tuberculous osteomyelitis runs a chronic, insidious course as in the patient presented. The most common clinical manifestation is localised pain and swelling that may precede signs of inflammation and radiological changes by weeks or months. Discharging sinus, such as the ulcer seen in the patient presented, may develop in chronic cases. Constitutional symptoms such as fever and weight loss are uncommon. ${ }^{125}$

Diagnosis of tuberculous osteomyelitis is usually delayed because the signs and symptoms are subtle. Laboratory tests, like erythrocyte sedimentation rate, complete blood count and joint fluid aspiration are inconclusive most of the time. ${ }^{6}$ Moreover, the radiographic appearance of tuberculous osteomyelitis depends on the stage of disease on presentation. There may be no radiolgical change initially except mild soft tissue swelling. In advanced stage, it may mimic chronic pyogenic osteomyelitis, tumours or granulomatous lesions. The typical radiological appearance of tuberculous osteomyelitis is a small focal area of osteolysis located eccentrically with the presence of local osteopenia as in the patient presented. However, the occurrence of typical radiological appearance is uncommon. ${ }^{1}$

To make a definite diagnosis; a high index of clinical suspicion and open biopsy for bacteriological and histological examination is needed. For the patient reported, the pulmonary lesion raised the possibility of tuberculosis osteomy- elitis in his 5th metatarsal lesion, which was confirmed by histological examination as well as bacteriological culture. However, chest radiography is an unreliable tool to aid diagnosis as only around $15 \%-20 \%$ of patients had concomitant active pulmonary lesion. ${ }^{13}$

As the subtle nature of tuberculosis might distract the emergency physician from the diagnosis, we should maintain a high index of suspicion of tuberculous osteomyelitis when patients, especially the elderly and the immunocompromised, present with chronic musculoskeletal symptoms not responding to the usual treatment.

M C Yuen would like to thank Dr Y P Li for his helpful advice in the preparation of this report.

Contributors

M C Yuen is the senior medical officer of the Accident and Emergency Department of Kwong Wah Hospital. W K Tung is the chief of services and consultant of the Accident and Emergency Department of Kwong Wah Hospital. W K Tung initiated the idea of writing this case report. M C Yuen collected the case history, reviewed the literature, and wrote up the report. M C Yuen is the guarantor of this report.
Yistory, reviewed the literature, and

1 Vohra R, Kang HS, Dogra S, et al. Tuberculosis osteomyelitis. F Bone foint Surg Br 1997;79:562-6.

2 Watts HG, Lifeso RM. Tuberculosis of bones and joints. 7 Bone foint Surg Am 1996;78:288-98.

3 Vallejo JG, Ong LT, Starke JR. Tuberculous osteomyelitis of Vallejo JG, Ong LT, Starke JR. Tuberculous osteomyelitis of
the long bones in children. Pediatr Infect Dis f 1995;14:5246.

4 Huang $\mathrm{CH}$. Extra-articular tuberculous osteomyelitis. A report of 11 cases. Int Orthop 1996;20:169-71.

5 Garcia-Porrua C, Gonzalez-Gay MA, Sanchez-Andrade A, et al. Arthritis in the right great toe as the clinical presentation of tuberculosis. Arthritis Rheum 1998;41:374-5.

6 Wallace R, Cohen AS. Tuberculous arthritis: a report of two cases with review of biopsy and synovial fluid findings. $\mathrm{Am}$ $\mathcal{f}$ Med 1976;61:277-82. 